\title{
Extended Spectrum Beta-Lactamase Production in Uropathogens Isolated from Hospitalized Patients with Chronic Pyelonephritis
}

\author{
Olga I. Chub ${ }^{*}, 1$, Aleksandr V. Bilchenko ${ }^{1}$ and Igor Khalin ${ }^{2}$ \\ ${ }^{1}$ Kharkiv Medical Academy for Postgraduate Education, Ukraine \\ ${ }^{2}$ National Defense University of Malaysia, Faculty of Medicine and Defense Health, Malaysia
}

\begin{abstract}
Background: Increased multidrug resistance of extended-spectrum beta-lactamases (ESBLs) compromises the efficacy of treatment of urinary tract infections.

Objective: The objective of this study is to determine the prevalence of ESBL-producing uropathogens from hospitalized patients with chronic pyelonephritis and to identify the presence of genes involved in the resistance.

Methods: A cross-sectional study of 105 patients with chronic pyelonephritis, treated in Kharkiv City Clinical Emergency Hospital, Ukraine was carried. Bacterial isolates were collected, antimicrobial susceptibility of isolates was determined by the Kirby Bauer disk diffusion method and screening for the presence of blaSHV, blaTEM, blaCTX-M ESBL genes was performed by polymerase chain reaction.

Results: 84 (80\%) patients had positive urine cultures. Escherichia coli was the most common microorganism isolated. Among them, $29(25.2 \%)$ were found to be ESBL producers. Out of 53 E. coli isolates, $10(18.9 \%), 4(7.5 \%)$ and 6 $(11.3 \%)$ were identified to carry bla(TEM), bla(SHV) and bla(CTX-M) beta-lactamase genes, respectively. The highest resistance was observed against ampicillin (75.9\%), ciprofloxacin (48.3\%), levofloxacin (41.4\%) and gentamicin (41.4\%). Beside this, only meropenem (96.6\% susceptibility), nitroxolinum $(86.2 \%)$ and fosfomycin (72.4\%) exhibited a good enough activity against ESBLs-producing urinary strains.
\end{abstract}

Conclusion: Isolation and detection of ESBL-producing strains are essential for the selection of the most effective antibiotic for the empiric treatment.

Keywords: Beta-lactams, chronic pyelonephritis, extended-spectrum beta-lactamases, plasmids, resistance.

\section{INTRODUCTION}

Urinary tract infections (UTIs) are thought to be the most common group of bacterial infections worldwide [1,2]. In Ukraine, the proportion of chronic pyelonephritis episodes remains at a high level reaching $40.7 \%$ in 2010 ; moreover, the morbidity and prevalence among general patients with chronic kidney disease has also increased to alarming values $40743(87.2 / 100,000$ population) and 381772 (816.6/100,000 population) respectively [3]. Besides this, more than 100,000 hospital admissions per year in the U.S. are UTI patients, among which patients with pyelonephritis are the most frequent. As for US outpatients, approximately $15 \%$ of antibiotics used are prescribed for UTIs [4].

In fact, Escherichia coli is considered to be a key bacteria in the pathogenesis of UTIs [5]. Meanwhile, beta-lactams and fluoroquinolones are well-known as the main therapeutics effective to treat such infections ${ }^{4}$. However, recent studies in Europe and the United States have demonstrated a steady increase in the rate of uropathogen resistance to commonly prescribed antibiotics, and this

*Address correspondence to this author at the Kharkiv Medical Academy for Postgraduate Education, Ukraine; Tel: +380953545759;

Fax: +3 (8057) 711-80-25; Email: o.chub@mail.ru obviously will lead to a reduction in therapeutic possibilities of UTI [6].

According to the published data, acquired resistance to beta-lactams is predominantly mediated by extendedspectrum beta-lactamases (ESBLs) that compromise the efficacy of all known beta-lactams, except cephamycine and carbapenems [5]. This can occur due to hydrolysis of the beta-lactam ring and may be obstructed by $\beta$-lactamase inhibitors such as clavulanic acid [7]. This type of resistance is basically encoded by plasmids derived from TEM or SHV family; however, the CTX-M type of ESBLs has become the most common over the past decade [5]. ESBLs are often encoded by genes located on large plasmids, which also carry genes related with resistance to other antimicrobial agents such as aminoglycosides, trimethoprim, sulphonamides, tetracyclines and chloramphenicol [7]. Alongside this, many studies have reported fluoroquinolone resistance mediated by co-transfer of the $q n r$ determinant on ESBL-producing plasmids [8,9]. Thus, multi-drug resistance (MDR) appears to be a fairly frequent characteristic of ESBL-producing enterobacterial isolates, and therefore ESBL-producing organisms pose a major challenge for clinicians, limiting therapeutic options [10].

Actually, data collected from European and intercontinental surveillance studies regarding the ESBL- 
producing Enterobacteriaceae, have consistently shown variable proportions among the different geographic locations, enterobacterial species and isolates from different sources (Table 1). In addition, it has been reported by the European Antimicrobial Resistance Surveillance Studies (EARSS) that the level of ESBL-positivity among Escherichia coli isolates resistant to third-generation cephalosporins fluctuates from $85 \%$ to $100 \%$ [5]. Furthermore, SENTRY Antimicrobial Surveillance Program has demonstrated that ESBL-phenotype rate among Klebsiella spp. rose by $41.8 \%$ in 2011 , compared to only $27.5 \%$ in 2009 [11]. The above-mentioned studies cover the patterns of urinary pathogen resistance in several parts of the world fairly well; however, similar data collection has yet to be done in the region of Eastern Europe. We did not find any publications regarding ESBL-producing prevalence in Ukraine as well as in contiguous countries, like Belarus, Moldova or Russia. Therefore, the aim of our cross-sectional study is to determine the prevalence of ESBL-producing uropathogens from hospitalized patients with chronic pyelonephritis, treated in the Kharkiv City Clinical Emergency Hospital, Ukraine and to identify the presence of genes involved in the resistance, specifically bla(TEM), bla(SHV) and bla(CTX-M).

\section{SUBJECTS AND METHODS}

\section{Bacterial Isolates}

The study was carried out between April 2013 and February 2014. Midstream urine from the patients with chronic pyelonephritis was collected in a sterile container and processed in the medical biology department of the Kharkiv City Clinical Emergency Hospital within 2 hours of collection. Urine samples were inoculated on blood agar or chromogenic media ChromID CPS (bioMerieux, France) then positive cases were incubated at $37{ }^{\circ} \mathrm{C}$ for 24 hours, and negative cases at $37{ }^{\circ} \mathrm{C}$ for 48 hours. Samples were considered significant if the number of colony forming units (CFU) was $\geq 10 \mathrm{CFU} / \mathrm{ml}$ of urine. The bacteria that had grown with significant counts were subjected to further investigation, namely their colony morphologies, Gram's smears, motilities and biochemical reactions were evaluated.

\section{Antimicrobial Susceptibility Testing}

The antimicrobial susceptibility of isolates was determined by the Kirby Bauer disk diffusion method on Mueller-Hinton agar-containing plates. The size of zone around each antimicrobial disk was interpreted as sensitive, intermediate or resistant [12]. The following antibiotics were tested: ampicillin, amoxicillin/clavulanate, ceftriaxone, cefepime, ciprofloxacin, levofloxacin, nitroxolinum, furamag, amikacin, gentamicin, nitrofurantoin, meropenem ("Limited Liability Company ASPECT", Kyiv, Ukraine) and co-trimoxazole, furagin, fosfomycin (HIMEDIA Laboratories, Pvt. Ltd., Mumbai, India).

\section{Detection of Beta-Lactamase Genes}

The total DNA extraction was performed for all samples using the heat-shock technique [13]. Screening for the presence of blaSHV, blaTEM, blaCTX-M ESBL genes was performed by polymerase chain reaction (PCR) sequencing assays. The following primers: bla $a_{T E M}$ (5'-ATG AGT ATT CAA CAT TTC CG; 5'-CCA ATG CTT AAT CAG TGA GG); $b l a_{S H V}$ (5'-ATG CGT TAT ATT CGC CTG TG; 5'AGC GTT GCC AGT GCT CGA TC); bla $a_{C T X-M}$ (5'-SCS ATG TGC AGY ACC AGT AA; 5'-ACC AGA AYV AGC GGB GC) had been explored. PCR products were analyzed by agarose gel electrophoresis and stained with ethidium bromide. Plasmid DNA, used as a molecular weight marker, was hydrolyzed by the enzyme puc19 HpaII [13].

\section{Ethics}

Each patient was aware about the data collection, and written informed consent was obtained from each subject. The study protocol has been approved by the ethics committee at the Kharkiv Medical Academy for Postgraduate Education, Kharkiv, Ukraine (No. 2, 22.02.2013). Anonymity has been guaranteed during and after the study.

\section{RESULTS}

Out of 105 adult patients with chronic pyelonephritis (the diagnosis chronic pyelonephritis has been stated in accordance with the criteria established by European Association of Urologists [4]), treated in Kharkiv City Clinical Emergency Hospital, Ukraine 84 (80\%) patients had positive urine cultures. Among those, 6 (7.1\%) were male and $78(92.9 \%)$ were female. From them, 115 different microorganisms were isolated, where 34 (29.6\%) were grampositive and $81(70.4 \%)$ were gram negative bacterial strains. The majority of the isolates $(n=73)$ were retrieved from patients between ages 18-65 years, while 42 isolates were obtained from those aged more than 65 years. All patients were discharged from the hospital; no mortality case had been reported during hospitalization.

Overall, Escherichia coli was the most common microorganism isolated $(53 / 115,46.1 \%)$, while, among the

Table 1. Global surveillance studies including ESBL-producing bacterial isolates.

\begin{tabular}{|c|c|c|c|c|}
\hline SENTRY [11] & 2009-2011 & Blood, urine, respiratory tract & $16.6 \%$ & $41.8 \%$ \\
\hline SMART [15] & 2002-2011 & Intra-abdominal, urinary tract & $11.8 \%$ & $17.9 \%$ \\
\hline
\end{tabular}

*ESBL-positive isolates among isolates resistant to third-generation cephalosporins. 
Table 2. Distribution of pathogens in urine isolates according to age and gender.

\begin{tabular}{|c|c|c|c|c|c|}
\hline Organism & Total $(n=115)$ & Male $(n=6)$ & Female $(n=78)$ & $\leq 65$ yrs $(n=54)$ & $\geq 65$ yrs $(n=30)$ \\
\hline E. coli & $53(46.1)$ & $1(16.7 \%)$ & $52(66.7 \%)$ & $31(57.4 \%)$ & $22(73.3 \%)$ \\
\hline P. mirabilis & $8(6.9)$ & $0(0.0)$ & $8(10.3 \%)$ & $4(7.4 \%)$ & $4(13.3 \%)$ \\
\hline P. aeruginosa & $8(6.9)$ & $2(33.3 \%)$ & $6(7.7 \%)$ & $8(14.8 \%)$ & $0(0.0)$ \\
\hline Serratia spp. & $2(1.7)$ & $1(16.7 \%)$ & $1(1.3 \%)$ & $0(0.0)$ & $2(6.7 \%)$ \\
\hline Enterococcus spp. & $17(14.8)$ & $2(33.3 \%)$ & $15(19.2 \%)$ & $12(22.2 \%)$ & $5(16.7 \%)$ \\
\hline Staphylococcus spp. & $12(10.4)$ & $0(0.0)$ & $12(15.4 \%)$ & $11(20.4 \%)$ & $1(3.3 \%)$ \\
\hline Corynebacterium & $4(3.5)$ & $0(0.0)$ & $4(5.1 \%)$ & $0(0.0)$ & $4(13.3 \%)$ \\
\hline
\end{tabular}

gram-positive bacterial, Enterococcus spp. and Staphylococcus spp. were the dominant pathogens strains. The distribution of pathogens according to patient age and gender is shown in Table 2. Beside this, among 115 isolates, 29 (25.2\%) were found to be ESBL producers. Out of $53 \mathrm{E}$. coli isolates, 10 $(18.9 \%), 4(7.5 \%)$ and $6(11.3 \%)$ were identified to carry bla(TEM), bla(SHV) and bla(CTX-M) beta-lactamase genes, respectively. Of note, out of nine $K$. pneumoniae, two isolates $(22 \%)$ produced ESBL, carrying blaCTX-M. Four P. mirabilis strains carried bla(TEM), bla(CTX-M) and two bla(SHV) genes, while Serratia spp. and Staphylococcus spp. were positive for one bla(TEM) and bla(CTX-M), respectively. Overall, out of 29 ESBL-producing isolates, $6(20.7 \%)$ coharboured at least two different bla genes and TEM ESBLs were the most common (Table $\mathbf{3}$ ).

Table 4 demonstrates susceptibility of isolated strains against a spectrum of 15 selected antimicrobial agents of different classes. The highest resistance was observed against ampicillin (75.9\%), ciprofloxacin (48.3\%), third generation cephalosporins (45\%) levofloxacin $(41.4 \%)$ and gentamicin $(41.4 \%)$. Beside this, only meropenem $(96.6 \%$ susceptibility), nitroxolinum $(86.2 \%)$ and fosfomycin (72.4\%) exhibited a good enough activity against ESBLsproducing urinary strains. Interestingly, 17 (42.5\%) patients were experienced to use a beta-lactam antibiotic in the preceding year prior hospitalization. Of note, that $12(30 \%)$ of the ESBL-producers were isolated on the fifth day after the beginning of antibiotic therapy.

\section{DISCUSSION}

This is the first study demonstrating the prevalence of ESBL's genes among the uropathogens isolated from pyelonephritis patients in Ukraine. In this cross-sectional study we have shown that $29(25.2 \%)$ of urinary isolates were ESBL producers. It is clearly seen from other papers that prevalence of ESBL producers among clinical isolates varies from country to country. According to Annual Report of the European Antimicrobial Resistance Surveillance Network (EARS-Net) 2013, the prevalence of ESBL among clinical strains of $E$. coli and $K$. pneumoniae, resistant to third-generation cephalosporins, varied from $85 \%$ to $100 \%$ [5]. The presence of ESBL was confirmed in $35.1 \%$ of the $K$. pneumoniae isolates according to the results of the SENTRY Antimicrobial Surveillance Program in the United States [11]. The prevalence of ESBL producing isolates of $E$. coli in studied patients in Mashhad, Iran, was 33.3\%[14]. It has been also revealed in our study that TEM-type ESBLs was the most common with its prevalence of $41.4 \%$. The frequency of CTX-M and SHV types of ESBLs were $34.5 \%$ and $24.1 \%$ respectively.

Conventionally, the strains, which are ESBL producers, are also known as multidrug resistant organisms. They usually perform less susceptibility to beta-lactams as well as to other classes of antibacterials including trimethoprimsulfamethoxazole, fluoroquinolones and aminoglycosides. Moreover, high levels of resistance to ampicillin,

Table 3. Prevalence of ESBL and $\beta$-lactamase genes in isolates of urine.

\begin{tabular}{|c|c|c|c|c|}
\hline \multirow{2}{*}{ Uropathogens } & \multirow{2}{*}{ Total n (\%) } & \multicolumn{3}{|c|}{ ESBLs Genes n (\%) } \\
\cline { 3 - 5 } & & blaCTX-M & blaTEM & blaSHV \\
\hline \hline E. coli & $20(37.7)$ & $6(30)$ & $0(0.0)$ & $4(20)$ \\
\hline K. pneumoniae & $2(22.2)$ & $2(100)$ & $1(25)$ & $2(0.0)$ \\
\hline P. mirabilis & $4(50)$ & $1(25)$ & $1(100)$ & $0(0.0)$ \\
\hline Serratia spp. & $1(50)$ & $0(0.0)$ & $0(0.0)$ & $0(0.0)$ \\
\hline Staphylococcus spp. & $1(8.3)$ & $1(100)$ & $0(0.0)$ & $1(100)$ \\
\hline Corynebacterium & $1(25)$ & $0(0.0)$ & \\
\hline
\end{tabular}


Table 4. Antimicrobial susceptibility pattern of ESBL producing urinary isolates.

\begin{tabular}{|c|c|c|c|}
\hline Drug & Sensitive n (\%) & Intermediate n (\%) & Resistance n (\%) \\
\hline Ampicillin & $3(10.3)$ & $4(13.8)$ & $22(75.9)$ \\
\hline Ceftriaxone & $19(65.5)$ & $1(3.4)$ & $9(31)$ \\
\hline Cefotaxime & $10(34.5)$ & $2(6.8)$ & $17(58.7)$ \\
\hline Levofloxacin & $17(58.6)$ & $0(0.0)$ & $12(41.4)$ \\
\hline Co-trimoxazole & $19(65.5)$ & $1(3.4)$ & $9(31)$ \\
\hline Nitroxolinum & $25(86.2)$ & $1(3.4)$ & $3(10.3)$ \\
\hline Furamag & $17(58.6)$ & $4(13.8)$ & $8(27.6)$ \\
\hline Meropenem & $28(96.6)$ & $0(0.0)$ & $1(3.4)$ \\
\hline Furagin & $17(58.6)$ & $4(13.8)$ & $8(27.6)$ \\
\hline Fosfomycin & $21(72.4)$ & $0(0.0)$ & $8(27.6)$ \\
\hline
\end{tabular}

levofloxacin, ciprofloxacin and gentamicin has been reported in other studies worldwide [15-18]. In line with these data, our findings showed high resistance rates to ampicillin (75.9\%), amoxicillin/clavulanate $(41.4 \%)$, ciprofloxacin $(48.3 \%)$, levofloxacin $(41.4 \%)$, gentamicin (41.4\%).

In Ukraine, a study, regarding bacterial flora of the urinary tract of patients with pyelonephritis and sensitivity/ resistance of isolated pathogens to antibiotics, had been conducted in 2010 [19]. We found that $23 \%$ of isolated strains were resistant to more than 10 antimicrobials. With that, high level of resistance was observed in $E$. coli to penicillins (49\%), tetracyclines (40-49\%) and fluoroquinolones (17-32\%). For other pathogens, level of multidrug resistance to $K$. pneumoniae rose up to $20 \%$, P. mirabilis $14 \%$, and the highest resistance was shown to E. faecalis $50 \%$. However, the prevalence of ESBLs among resistant strains has never been studied.

Thus, in Ukraine, the antimicrobial resistance rates have been substantially increasing among urinary strains over the last years. The most noticeable increases were observed to penicillins (from $49 \%$ in 2010 to $75,9 \%$ in 2014) and in fluoroquinolones (from $17-32 \%$ in 2010 to $48.3 \%$ in 2014). Additionally, susceptibility to third generation cephalosporins has dramatically dropped from $92-100 \%$ in 2010 to $55 \%$ in 2014 . In contrast, only meropenem $(96.6 \%$ susceptible) and nitroxolinum (86.2\%) exhibit good activity against ESBL-producing strains from hospitalized patients with pyelonephritis.

\section{CONCLUSION}

The prevalence of ESBLs among uropathogens, isolated from hospitalized patients with pyelonephritis is $25.2 \%$. TEM-type of ESBLs is the most common isolated gene. In this study, $96.6 \%$ of the ESBL producing isolates are identified as meropenem susceptible. Nitroxolinum and fosfomycin are also found to be highly effective drugs in vitro.

Taking all these things into consideration, we can assume that the isolation and detection of ESBL-producing strains are essential for the selection of the most effective antibiotic for empiric treatment. Since most of ESBL genes are carried by plasmids, these genes could easily transfer among hospitalized patients. This is a major factor for increasing spread of ESBL producers. Therefore, the rational use of antibiotics in practice and/or the proper identification of ESBL-producing bacteria in communities are crucial for further prevention of antimicrobial resistance development.

\section{CONFLICT OF INTEREST}

The authors confirm that this article content has no conflict of interest.

\section{ACKNOWLEDGEMENTS}

Declared none.

\section{REFERENCES}

[1] Foxman B. Urinary tract infection syndromes: occurrence, recurrence, bacteriology, risk factors, and disease burden. Infect Dis Clin North Am 2014; 28(1): 1-13.

[2] Eurosurveillance editorial team. ECDC publishes the annual epidemiological report 2012. Euro Surveill. 2013; 18(10): pii=20418. Available online: http:/www.eurosurveillance.org/Vie wArticle.aspx?ArticleId=20418

[3] Kolesnik MO, Stepanova NM, Lebid LO, et al. Adapted clinical instruction for the better practice of diagnosis, treatment and prevention of urinary tract infections in women. Ukr J Nephrol Dial 2012; 2(34): 53-77.

[4] Grabe, M, Bartoletti R, Cai T, et al. Guidelines on urological infections. European Association of Urology 2014; pp. 1-108. 
[5] European Centre for Disease Prevention and Control. Antimicrobial resistance surveillance in Europe 2012. Annual Report of the European Antimicrobial Resistance Surveillance Network (EARS-Net). Stockholm: ECDC; 2013. Available from: http://ecdc.europa.eu/en/publications/Publications/antimicrobialresistance-surveillance-europe-2012.pdf

[6] Coque T M, Baquero F, Canton R. Increasing prevalence of ESBL - producing Enterobacteriaceae in Europe. Euro Surveill 2008; 13 (47): 20.

[7] Rawat D, Nair D. Extended-spectrum $\beta$-lactamases in gram negative bacteria. J Glob Infect Dis 2010; 2(3): 263-74.

[8] Mammeri H, Van De Loo M, Poirel L, et al. Emergence of plasmid-mediated quinolone resistance in Escherichia coli in Europe. Antimicrob Agents Chemother 2005; 49: 71-6.

[9] Wang M, Sahm DF, Jacoby GA, et al. Emerging plasmid-mediated quinolone resistance associated with the qnr gene in Klebsiella pneumoniae clinical isolates in the United States. Antimicrob Agents Chemother 2004; 48: 1295-9.

[10] Crémet L, Caroff N, Dauvergne S, Reynaud A, Lepelletier D, Corvec S. Prevalence of plasmid-mediated quinolone resistance determinants in ESBL Enterobacteriaceae clinical isolates over a 1 year period in a French hospital. Patho Bio 2011; 59(3): 151-6.

[11] Sader HS, Farrell DJ, Flamm RK, et al. Antimicrobial susceptibility of Gram-negative organisms isolated from patients hospitalized in intensive care units in United States and European hospitals (2009-2011). Diagn Microbiol Infect Dis 2014; 78(4): 443-8.

[12] Performance standards for detection of antimicrobial susceptibility of microorganisms. Ministry of Health, Ukraine. Normative document. 2007. Available from: http://mozdocs.kiev.ua/view.php $? \mathrm{id}=6958$

[13] Sundsfjord A, Sundsfjord A, Simonsen GS, Haldorsen BC. Genetic methods for detection of antimicrobial resistance. DAHL APMIS 2004; 112: 815-37.

[14] Nakhaei Moghaddam M, Forghanifard MM, Moshrefi S. Prevalence and molecular characterization of plasmid-mediated extended spectrum $\beta$-lactamase genes (blaTEM, blaCTX and blaSHV) among urinary Escherichia coli clinical isolates in Mashhad, Iran. Iran J Basic Med Sci 2012; 15(3): 833-9.

[15] Morrissey I Hackel M, Badal R, et al. A review of ten years of the study for monitoring antimicrobial resistance trends (SMART) from 2002 to 2011. Pharmaceuticals 2013; 6: 1335-46.

[16] Balode A, Punda-Polić V, Dowzicky MJ et al. Results from the tigecycline evaluation and surveillance trial (T.E.S.T.) 2004-2010. Int J Antimicrob Agents 2013; 41(6): 527-35.

[17] Gibold L, Robin F, Tan RN, et al. Four-year epidemiological study of extended-spectrum $\beta$-lactamase-producing Enterobacteriaceae in a French teaching hospital. Clin Microbiol Infect 2014; 20(1): 20-6.

[18] Huh K, Kim J, Cho SY, et al. Continuous increase of the antimicrobial resistance among gram-negative pathogens causing bacteremia: a nationwide surveillance study by the Korean Network for Study on Infectious Diseases (KONSID). Diagn Microbiol Infect Dis 2013; 76(4): 477-82.

[19] Kolesnyk MO. Spectrum of bacterial microflora of the uro-genital tract in patients with pyelonephritis and its antibiotic sensitivity.Ukrainian Journal of nephrology and dialysis 2010; 4(28): 5-10.

(C) Chub et al.; Licensee Bentham Open.

This is an open access article licensed under the terms of the Creative Commons Attribution Non-Commercial License (http://creativecommons.org/licenses/by-nc/3.0/) which permits unrestricted, non-commercial use, distribution and reproduction in any medium, provided the work is properly cited. 